\title{
Oxidative Desulfurization of benzothiophene derivatives with cis-dioxomolybdenum(VI) catalyst precursors, under extractive conditions
}

\author{
Francesco Ferella, ${ }^{\mathrm{a}}$ Lorenzo Biancalana ${ }^{\mathrm{b}, \mathrm{c}}$ Fabio Marchetti, ${ }^{\mathrm{b}, \mathrm{c},}$ Marcello Crucianellia, ${ }^{\mathrm{a}, *}$ \\ ${ }^{a}$ Department of Physical and Chemical Sciences, University of L'Aquila, via Vetoio, I-67100 L'Aquila, Italy \\ ${ }^{b}$ Department of Chemistry and Industrial Chemistry, University of Pisa, via G. Moruzzi 13, I-56124 Pisa, Italy \\ ${ }^{\mathrm{c} C I R C C}$, via Celso Ulpiani 27, I-70126 Bari, Italy
}

\begin{abstract}
The study of the activity of a series of heterogeneous cis-dioxomolybdenum(VI) based catalyst precursors for the hydrogen peroxide promoted oxidation of benzothiophene, 2- and 3methylbenzothiophene, dibenzothiophene and 4,6-dimethyldibenzothiophene, has been carried out. The last, chosen as model substrates of the common sulfurated derivatives of fuels, have been oxidized in the presence of selected imidazolium based ionic liquids, under extractive oxidative desulfurization conditions. The reactivity of sulfur-containing compounds toward the oxidation to their corresponding sulfones has been evaluated, firstly, for each type of substrate alone, and then applying the optimized procedure to a system formed by all sulfurated substrates. Our aim was to mimic the oxidation of a model diesel oil, under either thermal or microwave assisted heating. Cisdioxomolybdenum(VI) compounds behaved as very active and stable catalytic systems retaining, in one selected case, the original activity at least for three cycles. Imidazolium ionic liquids played a dual fundamental role not only as extractant and reaction medium but also as stabilizing system for both the oxidant and the catalytic agents. Under optimized microwave-assisted conditions, the quantitative elimination of sulfurated substrates from the model diesel oil, was obtained after only 1

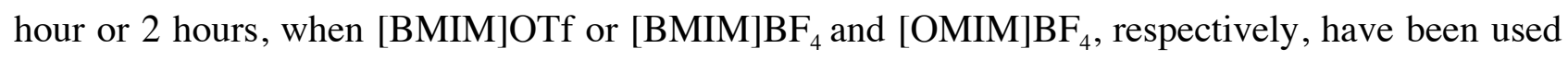
as ionic liquids.
\end{abstract}

Keywords: cis-dioxomolybdenum; oxidative desulfurization; heterogeneous catalysis; hydrogen peroxide; ionic liquids; microwave assisted oxidation

*Corresponding authors: Tel:: (+39) 0862-433308; e-mail: marcello.crucianelli@univaq.it (M. Crucianelli). Tel.: (+39) 050-2219219; e-mail: fabio.marchetti1974@unipi.it (F. Marchetti). 


\section{Introduction}

Organosulfur are undesirable compounds, when contained inside fuels, due to the negative environmental impact caused by $\mathrm{SO}_{\mathrm{x}}$ emissions during their combustion. As a consequence, removal of sulfur is becoming a worldwide challenge. Hazardous emissions of $\mathrm{SO}_{\mathrm{x}}$ are responsible for several undesired issues, such as acidic rain, air pollution, respiratory disorders as well as irreversible catalyst poisoning. To protect the environment against $\mathrm{SO}_{\mathrm{x}}$ pollution, several stringent environment legislations have been published to limit the sulfur content of fuels. For gasoline, the new standard of sulfur content may be restrained, in most countries, to less than $10 \mu \mathrm{g} / \mathrm{mL}$ [1]. Thus, the deep desulfurization of gasoline has become an important research topic [2]. The hydrodesulfurization (HDS) process achieves limited performances in the case of refractory Scontaining aromatic compounds, such as thiophene and substituted benzothiophenes (BTs), which require highly energy-demanding conditions (high temperature and pressure conditions). Several alternative and/or complementary desulfurization techniques to the conventional HDS have been proposed [3], among others, catalytic and biocatalytic oxidative desulfurization (ODS) or the combined technique based on extractive and catalytic oxidative desulfurization (ECODS) [4]. Frequently, transition metal containing nanocatalysts, with dimensions of less than $100 \mathrm{~nm}$, have been used in ODS processes in the last years, combining the advantages from the role played by the physical form of the catalyst in synergy with the chemical properties of the metal (or metals) species embedded in the system [5].

The oxidative desulfurization process is based on the oxidation of the organosulfur compounds to the corresponding sulfoxides and/or sulfones which are highly polar and can thus be readily removed by adsorption, distillation, or solvent extraction.

Under ECODS conditions, the extraction of sulfur-containing products and the chemical or catalytic oxidation of sulfur compounds occur simultaneously in a one-pot biphasic system. Ionic liquids (ILs) are promising solvents and extractants for ECODS processes due to their excellent ability to solubilize inorganic/organic compounds, high thermal stability, low vapor pressure, and recyclability [6]. As largely accepted, catalyst immobilization is not the only benefit deriving from the use of ILs in catalysis. Of greater importance are a series of further positive issues like: the catalysts often become more reactive in the presence of ILs and, in some cases, the reactive catalytic species or reaction intermediates can be stabilized inside ILs, which often results in a significant improvement in the catalytic performance or even making it possible to achieve catalytic reactions that are not possible to conduct in common organic solvents [7]. The combination of the 
extraction and catalytic oxidation under ECODS process, using ILs as solvents, has been proven to be more efficient compared to the mere ILs extraction.

Sulfur removal under environmental friendly oxidative conditions requires the use of green stoichiometric oxidants, mainly molecular oxygen $\left(\mathrm{O}_{2}\right)$ [8] or hydrogen peroxide $\left(\mathrm{H}_{2} \mathrm{O}_{2}\right)$ [9]. The latter is, in effect, the most attractive oxidant to apply in ODS reactions due to its low cost, easy handling and ready availability, high oxygen content and ecological compatibility, yielding only water as by-product. Many oxidation catalysts, such as tungsten- or molybdenum-containing polyoxometalates (POMs) [10], titanium supported on silica materials [11], and homogeneous or heterogeneous oxometal complexes [12] have been successfully applied in ODS processes.

In particular, $\mathrm{Mo}(\mathrm{VI})$ compounds are able to activate mild oxidants such as $\mathrm{H}_{2} \mathrm{O}_{2}$ and alkyl hydroperoxides, thus overcoming the severe environmental and health restrictions connected with the use of peracids [13]. On the other hand, oxomolybdenum(VI) complexes are well-known as catalysts or catalyst precursors for sulfur oxidation [14], even if their use as (pre)catalysts in ECODS processes, based on the use of ILs, has not yet been largely explored [12b,12e].

To further investigate the potential applicability of heterogeneous oxomolybdenum(VI) complexes toward the selective ECODS processes, here we report the study of a series of cisdioxomolybdenum(VI) containing catalyst precursors I-V (Chart 1) for the oxidation of benzothiophene (BT), 2- and 3-methylbenzothiophene (2Me-BT and 3Me-BT), dibenzothiophene (DBT) and 4,6-dimethyldibenzothiophene (DMDBT), as model substrates of the common sulfurated derivatives of fuels, in the presence of imidazolium based ILs (Chart 2). The best results we obtained during the optimization of the ECODS process, firstly for each type of sulfurated substrate alone, and then applying the optimized procedure to a model diesel oil under, either, thermal or microwave heating, will be described.

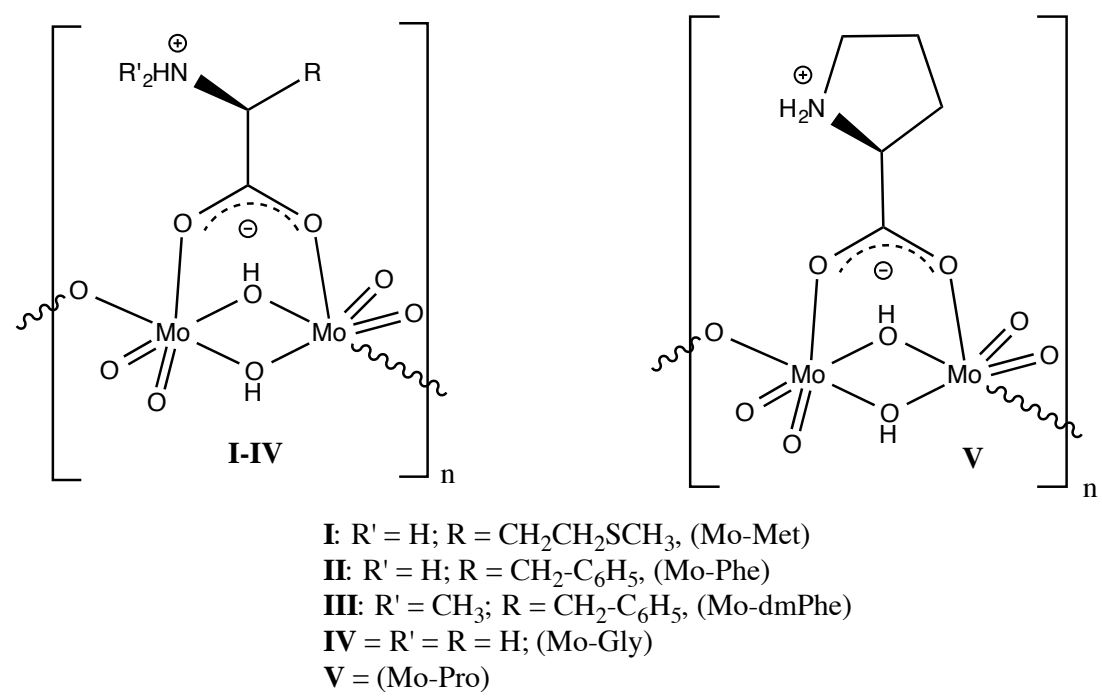


Chart 1. Structure of the Molybdenum(VI) complexes bearing L-Methionine (I, Mo-Met), L-Phenylalanine (II, MoPhe), N,N-dimethyl-L-Phenylalanine (III, Mo-dmPhe), Glycine (IV, Mo-Gly) and L-Proline (V, Mo-Pro) as $\alpha$-amino acid ligands, used in this work.
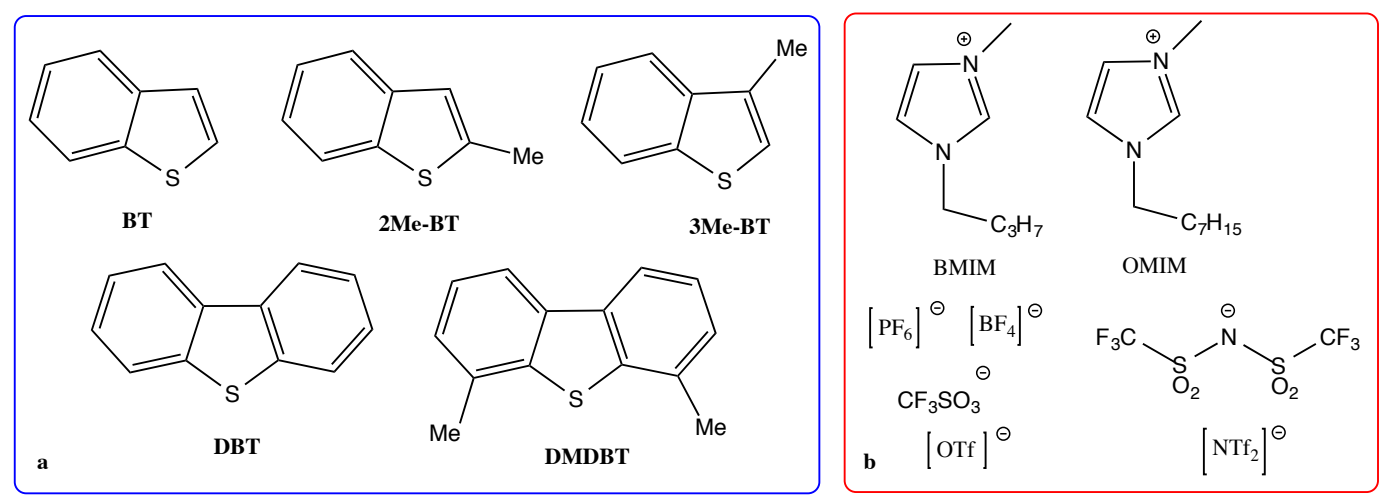

Chart 2. Structures of benzothiophene (BT) and its derivatives (part a) with cations and anions of imidazolium based ILs (part b), used in this work.

\section{Experimental Section}

\subsection{Materials and Methods}

All reagents (DBT, DMDBT, BT, 2-MeBT, 3Me-BT) and solvents were purchased from SigmaAldrich (Italy) in the highest purity grade available and were used as such. All ionic liquids, namely

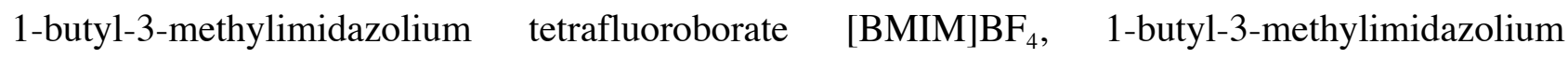
hexafluorophosphate $\left[\mathrm{BMIM}_{\mathrm{PF}_{6}}, 1\right.$-octyl-3-methylimidazolium tetrafluoroborate $[\mathrm{OMIM}] \mathrm{BF}_{4}, 1$ octyl-3-methylimidazolium hexafluorophosphate $\quad\left[\mathrm{OMIM}_{\mathrm{PF}_{6}}, \quad\right.$ 1-butyl-3-methylimidazolium trifluoromethansulfonate [BMIM]OTf and 1-butyl-3-methylimidazolium bis(trifluoromethylsulfonyl)imide [BMIM]NTf ${ }_{2}$ were purchased from Iolitec (https://iolitec.de). A $5.5 \mathrm{M}$ solution in $n$-decane of tert-butyl hydroperoxide (TBHP), or a $50 \% \mathrm{w} / \mathrm{w}$ aqueous solution of $\mathrm{H}_{2} \mathrm{O}_{2}$, were used as primary oxidants. Compounds $\mathbf{I}-\mathbf{V}$ were prepared according to the literature [15]. NMR spectra were recorded, at 298 K, with a Bruker Avance III 400 MHz instruments. Solidstate infrared spectra were recorded on a Perkin Elmer SpectrumOne FT-IR spectrometer, equipped with a UATR sampling accessory. Microwave studies were performed with the Biotage ${ }^{\circledR}$ Initiator $^{+}$ microwave synthesizer. Gas chromatographic analyses (GC-FID) were performed by means of a Hewlett-Packard 6890 series instrument equipped with a flame ionization detector, using a $30 \mathrm{~m} \times$ $0.32 \mathrm{~mm} \times 0.25 \mu \mathrm{m}$ film thickness (crosslinked $5 \%$ phenylmethylsiloxane) column and chromatography grade helium as carrier gas. In GC calculations, all peaks amounting to at least 
$0.5 \%$ of the total products were considered. ${ }^{1} \mathrm{H}$ and ${ }^{13} \mathrm{C}$ NMR analyses of products were performed after purification, and compared with authentic samples.

\subsection{Optimization study for the ECODS with ionic liquid [BMIM]BF 4}

The catalytic experiments were carried out under atmospheric pressure in a $5 \mathrm{~mL}$ flask equipped with a magnetic stirring bar, immersed in a thermostatic oil bath at $50{ }^{\circ} \mathrm{C}$. In a typical experiment: $0.001 \mathrm{mmol}$ of the selected catalyst and $0.10 \mathrm{mmol}$ of sulfurated substrate were added to a 5:1 (v/v) mixture of $n$-octane/[BMIM]BF 4 (1.2 mL), under stirring. The mixture was vigorously stirred for 5 min., at $50{ }^{\circ} \mathrm{C}$, and then added with 2.0 equiv. of $\mathrm{H}_{2} \mathrm{O}_{2}(50 \%$ aqueous solution) to start the oxidation process, at the same temperature. The progress of the oxidation was periodically monitored by withdrawing an aliquot $(3.0 \mu \mathrm{L})$ of the hydrocarbon phase, added with $3.0 \mu \mathrm{L}$ of $n$-hexadecane, as internal standard, then analyzed by GC-FID. When necessary, 1.0 additional equiv. of $\mathrm{H}_{2} \mathrm{O}_{2}$ was added at regular interval of time ( 1 hour), up to 4.0 eq. After 3 hours, the reaction was quenched by adding $1.0 \mathrm{~mL}$ of a $5 \%$ aqueous solution of $\mathrm{Na}_{2} \mathrm{~S}_{2} \mathrm{O}_{5}$ to the recovered organic phase, then the latter was extracted with $1.0 \mathrm{~mL}$ of chloroform, dried over anhydrous $\mathrm{MgSO}_{4}$ and analyzed by GC-FID. Control experiments performed with DBT in the absence of catalyst, otherwise under similar conditions, showed only poor substrate oxidation (less than 10\%).

\subsection{ECODS of the model diesel phase in selected ILs}

The catalytic experiments were carried out under atmospheric pressure in a $5 \mathrm{~mL}$ flask equipped with a magnetic stirring bar, immersed in a thermostatic oil bath at $50{ }^{\circ} \mathrm{C}$. In a typical experiment: the multicomponent model diesel phase was prepared by dissolving the proper amount (about 0.02 mmol) of each sulfurated substrate, namely DBT, DMDBT, BT, 2-MeBT and 3Me-BT, up to a total sulfur concentration of $2700 \mathrm{ppm}$, in $1.2 \mathrm{~mL}$ of a 5:1 (v/v) mixture of $n$-octane/IL, under stirring. The biphasic mixture, added with $0.001 \mathrm{mmol}$ of catalyst $\mathbf{I}$ was vigorously stirred for $5 \mathrm{~min}$., at 50 ${ }^{\circ} \mathrm{C}$, and then added with 2.0 equiv. of $\mathrm{H}_{2} \mathrm{O}_{2}(50 \%$ aqueous solution) to start the oxidation process, at the same temperature. The progress of the reaction was monitored as above described. 1.0 additional equiv. of $\mathrm{H}_{2} \mathrm{O}_{2}$ was added, at regular interval of time (1 hour), up to 5.0 eq. At the end of the reaction, we followed the same work-up procedure as above described.

\subsection{MW assisted ECODS of the model diesel phase in selected ILs}


The model diesel phase, prepared as above described, containing $0.001 \mathrm{mmol}$ of catalyst $\mathbf{I}$, was inserted into the appropriate MW glass vials $(0.5-2.0 \mathrm{~mL})$ with the stirring bar, added with 2.0 equiv. of $\mathrm{H}_{2} \mathrm{O}_{2}$ (50\% aqueous solution), and then sealed. The experiment was performed under control of the temperature $\left(90^{\circ} \mathrm{C}\right.$, power output average $\left.=200 \mathrm{~W}\right)$, by setting the following instrumental parameters: reaction running time $=28 \mathrm{~min}$., preceded by a prestirring of $2 \mathrm{~min}$; stir rate $=720 \mathrm{rpm}$; number of cycles $=4$ (2 in the case of $[\mathrm{BMIM}] \mathrm{OTf})$. The progress of the reaction was monitored as above described. 1.0 additional equiv. of $\mathrm{H}_{2} \mathrm{O}_{2}$ was added, after each cycle, up to 5.0 eq. At the end of the reaction, we followed the same work-up procedure as above described.

\subsection{Recycling experiments}

The recycling experiments were performed following the procedure reported on section 2.3, using

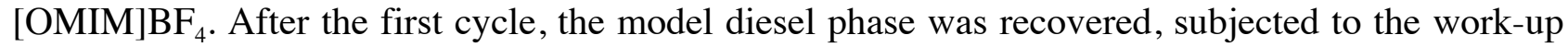
procedure reported on section 2.2, and analyzed by GC-FID to evaluate its S content. The residue of water was eliminated by evaporation of the IL phase, under reduced pressure. Then, new fresh portions of both model diesel and $\mathrm{H}_{2} \mathrm{O}_{2}$ were added to the recycled IL phase containing the entrapped catalyst, and a new cycle started. After the last cycle, the white solid formed was removed and analyzed by FT-IR.

\section{Results and Discussion}

\subsection{Optimization study}

We started with the evaluation of the activity, under ECODS conditions, of several selected benzothiophene derivatives, namely DBT, DMDBT, BT, 2Me-BT and 3Me-BT dissolved in $n$ octane, using [BMIM] $\mathrm{BF}_{4}$ as the water-miscible extracting medium. At the beginning, each starting material (s.m.) has been investigated with all the cis-dioxomolybdenum(VI) catalyst precursors I-V (Chart 1). We set up our starting experimental conditions, namely $n$-octane/IL ratio and temperature, according to several data available from literature [12c,16]. Briefly, $0.1 \mathrm{mmol}$ of the s.m. were dissolved in a $5.0 \mathrm{~mL}$ flask containing $0.001 \mathrm{mmol}$ of the selected catalyst, $1.0 \mathrm{~mL}$ of $n$ -

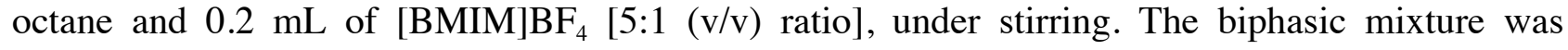
vigorously stirred for $5 \mathrm{~min}$., at $50{ }^{\circ} \mathrm{C}$, and then added with 2.0 equiv. of $\mathrm{H}_{2} \mathrm{O}_{2}(50 \%$ aqueous solution) to start the oxidation process. The progress of the oxidation was periodically monitored by 
withdrawing little amount of the hydrocarbon phase which was then analyzed by GC-FID. In order to force the oxidation of s.m. toward the corresponding sulfones, two additional equivalents of $\mathrm{H}_{2} \mathrm{O}_{2}$ were added (up to overall 4.0 equiv., after $1 \mathrm{~h}$ and $2 \mathrm{~h}$, respectively). After 3 hours, the reaction was quenched, and the percentage of the obtained conversion of s.m. are showed in Fig. 1.

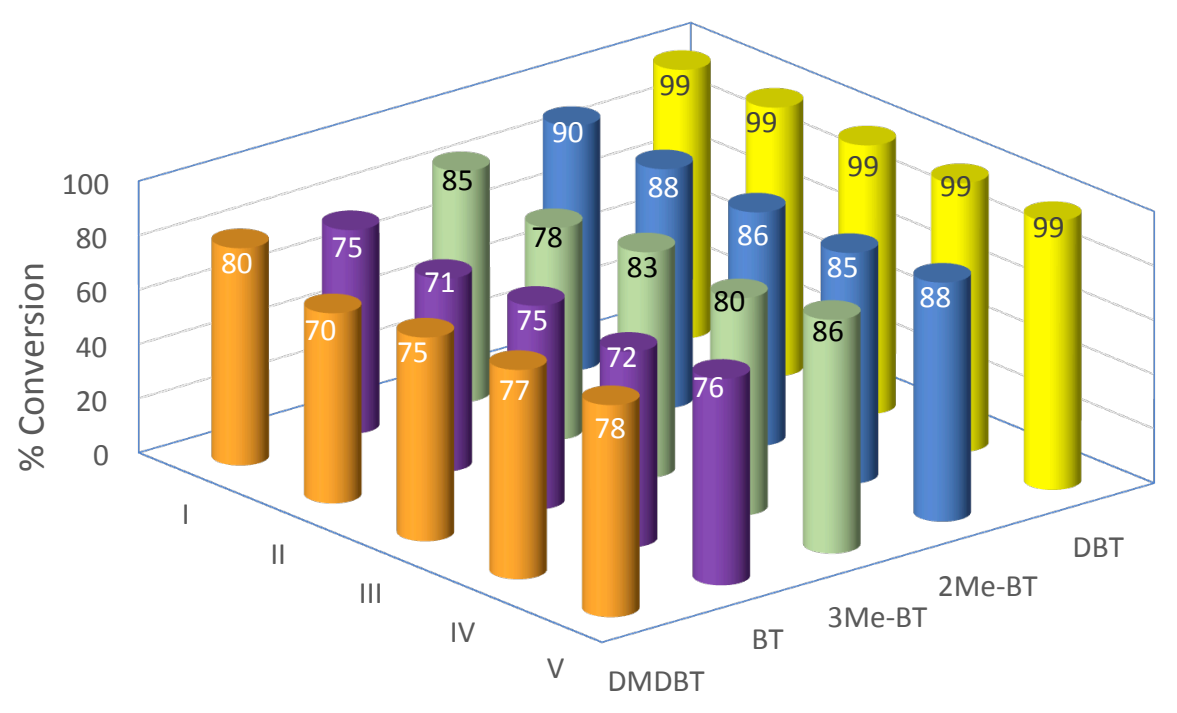

Figure 1. Percentage of conversion for the ECODS of sulfurated s.m. like DMDBT, BT, 3Me-BT, 2Me-BT and DBT in $n$-octane/BMIM-BF 4 (5:1 v/v), with catalysts I-V [(I, Mo-Met), (II, Mo-Phe), (III, Mo-dmPhe), (IV, Mo-Gly) (V, MoPro)], at $50^{\circ} \mathrm{C}$, for $3 \mathrm{~h}$.

All the different molybdenum catalysts (I-V) showed a very high activity, affording satisfactory conversion values of s.m., ranging from 70\% (DMDBT with catalyst II) to > 99\% (DBT for all catalysts), after only $3 \mathrm{~h}$, at $50{ }^{\circ} \mathrm{C}$. At the end of the reaction, in the case of less reactive s.m. like DMDBT and BT, only residual unreacted substrate was detected in the recovered hydrocarbon phase, being the corresponding more polar sulfones totally extracted by IL phase. In agreement with our previous study concerning olefins oxidation [17], no great differences in terms of catalytic activity between catalysts $\mathbf{I}-\mathbf{V}$, have been detected irrespective to the type of used s.m., slightly being catalyst $\mathbf{I}$ the more active.

On the basis of previous work on ECODS processes, a probable pathway for the oxidation of sulfurated substrates, under these conditions, is suggested for DBT and catalyst I in Scheme 1. 


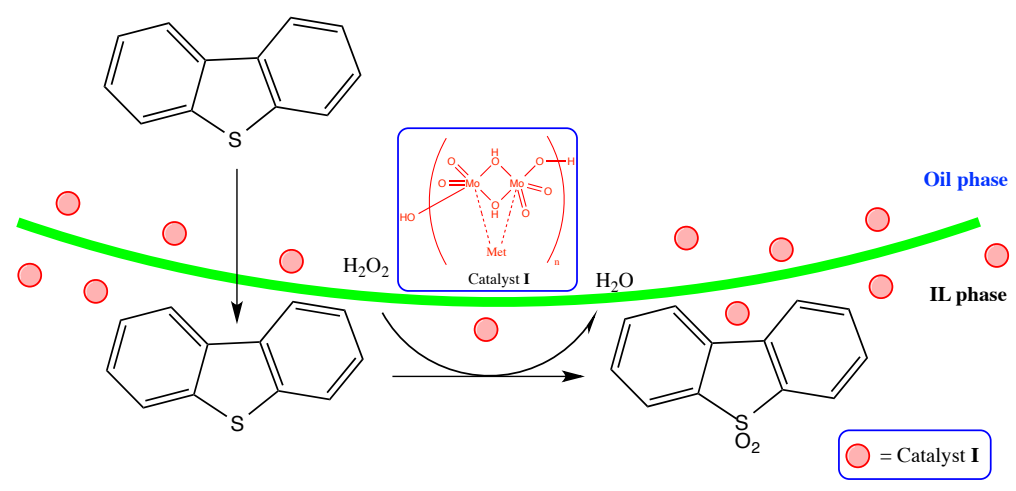

Scheme 1. Sketch of a plausible pathway for the ECODS of DBT, in $n$-octane/BMIM-BF ${ }_{4}$ system, with catalyst $\mathbf{I}$.

Initially, DBT was extracted from the hydrocarbon phase to IL phase, meanwhile being the heterogeneous catalyst I dispersed within the interphase of the solvent mixture. The oxidant is able to supply in a continuous way active oxygen to promote the formation of the catalytically active species, presumably a Mo(VI)-peroxo derivative [17]. Then, the latter promote the DBT oxidation toward the corresponding sulfone, within the IL phase. As a consequence, a continuous reduction of the initial concentration of DBT in the oil phase, is detected.

Blank runs (same conditions, without catalyst) were performed with DBT and, as expected, no significant substrate conversion (less than 10\%) was observed under otherwise similar conditions. Catalysts I-V behaved as totally insoluble, being dispersed at the middle layer of the solvent mixture, thus suggesting that we are working under real heterogeneous conditions. For these reasons, we decided to use a molar ratio s.m./catalyst $(\mathrm{S} / \mathrm{C})=100$, because lower amounts of catalyst gave unsatisfactory results for the overall efficiency of the process.

The order of reactivity showed by the different s.m., namely DBT $>2 \mathrm{Me}-\mathrm{BT} \geq 3 \mathrm{Me}-\mathrm{BT}>\mathrm{DMDBT}$ $>\mathrm{BT}$, may be rationalized either, considering the positive effect due to the increase of the electron density at the $\mathrm{S}$ atom, or bearing in mind the negative one caused by the increase of the steric hindrance influencing the accessibility at the same sulfur site $[16,18]$.

The ratio between $n$-octane/ILs is an important parameter to be evaluated in an ECODS process, mainly related to the high cost of ILs, but also considering the possible competitive depletion of precious components (aromatics and olefins) of a real diesel sample, depending on the extracting properties of ILs [19]. In order to minimize these drawbacks, thus making it interesting the use of ILs also for the potential industrial application of the ECODS process, it is necessary to keep low the dosage of IL. For these reasons, we studied the effect of the $[\mathrm{BMIM}] \mathrm{BF}_{4} / n$-octane volume ratio,

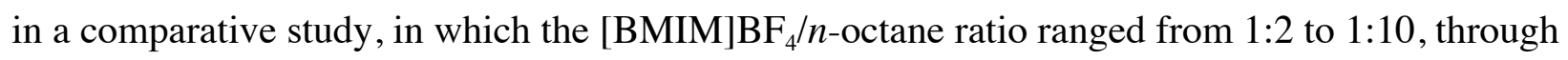
the $1: 5$ as the intermediate value. 


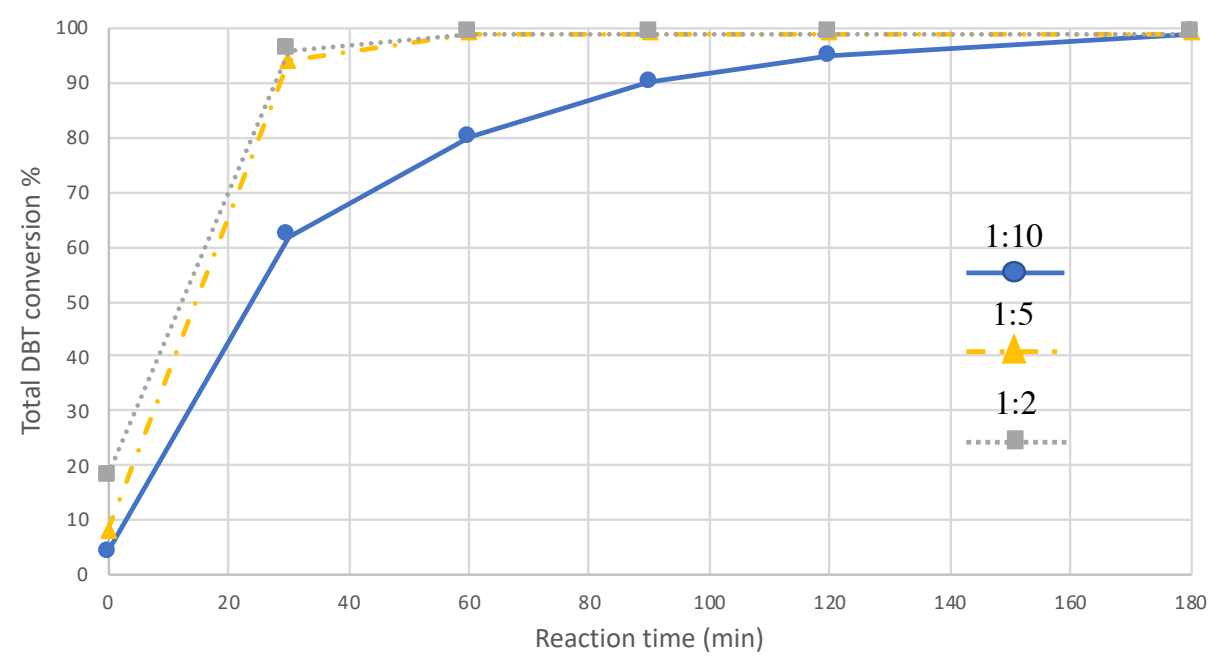

Figure 2. Total DBT conversion vs. reaction time with different $[\mathrm{BMIM}] \mathrm{BF}_{4} / \mathrm{n}$-octane volume ratio. Conditions: $\mathrm{T}=50$ ${ }^{\circ} \mathrm{C}, \mathrm{H}_{2} \mathrm{O}_{2}$ (2.0 equiv.), $0.1 \mathrm{mmol}$ of DBT, $1.0 \mathrm{~mol} \%$ of catalyst $\mathbf{I}$.

The experimental results showed in Fig. 2 prompted us to opt for the (5:1) n-octane/IL volume ratio as a good compromise, also considering that, even if the extraction of sulfones derived from DBT was higher at the beginning of the process for the higher ratio of IL, the efficiency of the overall ECODS reaction was similar for longer reaction times [12c]. On the other hand, the lower ratio of IL was inadequate, especially when working with the model diesel oil (see further).

For what it concerns the optimization of the reaction temperature, we worked at $50{ }^{\circ} \mathrm{C}$, because the experimental outcomes evidenced unsatisfying results working at lower temperature in terms of both total conversion of s.m. and of kinetic efficiency. Instead higher temperature, even if accelerating the kinetic of the oxidation, are responsible for the unproductive decomposition of the oxidant [20].

Having in mind to evaluate the role of different imidazolium based ILs on both, the efficiency of the extractive step and on the activation of the overall ECODS reaction, we studied the oxidation of the selected DBT in a series of $n$-octane/IL mixtures, containing different imidazolium based ILs

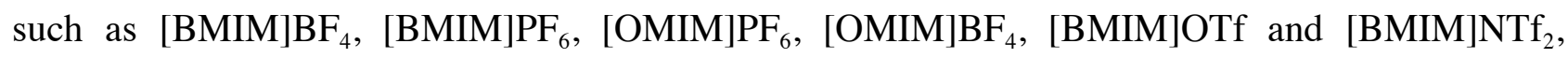
maintaining fixed the 5:1 n-octane/IL volume ratio, and using $\mathrm{H}_{2} \mathrm{O}_{2}$ or tert-butylhydroperoxide (TBHP, 70\% in decane) as oxidants. Since it is well known that TBHP has been largely used for oxidative catalysis with molybdenum complexes, we decided to evaluate its role as an alternative to $\mathrm{H}_{2} \mathrm{O}_{2}$, in view of the different water miscibility properties of the above described ILs. 


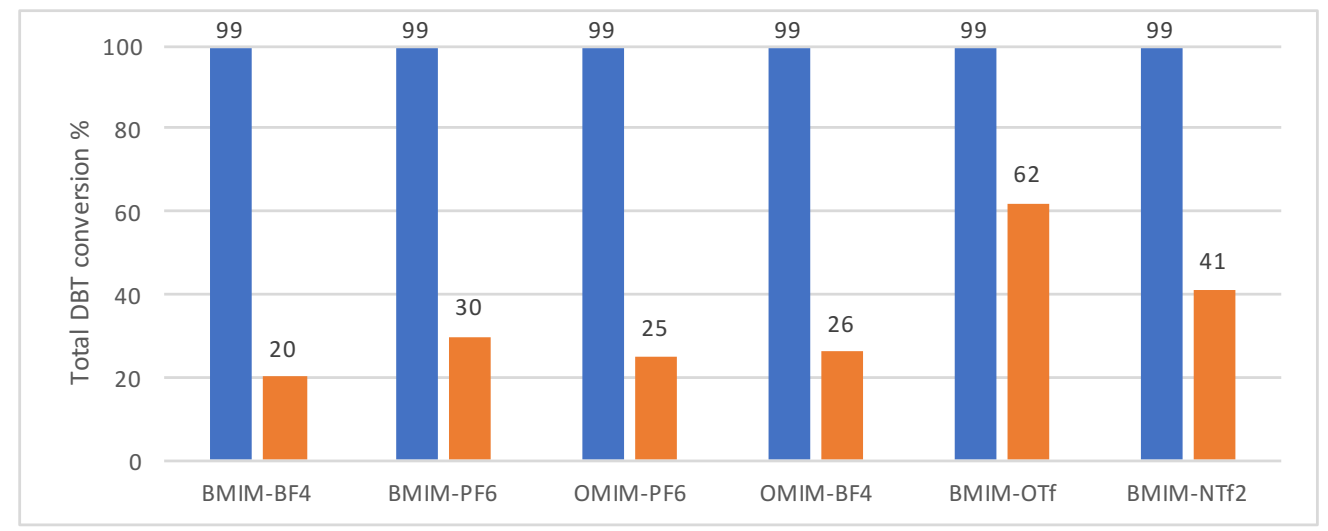

Figure 3. \% of DBT conversion, under ECODS, for a series of ILs with $\mathrm{H}_{2} \mathrm{O}_{2}$ (blue bar) or TBHP (orange bar) and catalyst $\mathbf{I}$.

The results reported in Figure 3 have been obtained, after 2 h, with catalyst $\mathbf{I}$ otherwise working under the same experimental conditions above described for the oxidation of the different sulfur substrates (Fig. 1). The reaction mixtures ( $n$-octane/IL $+\mathrm{H}_{2} \mathrm{O}_{2}$ ) were either biphasic (for the water

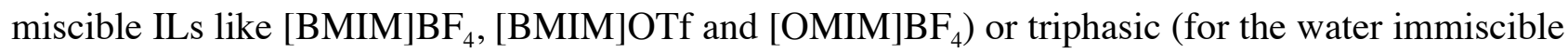

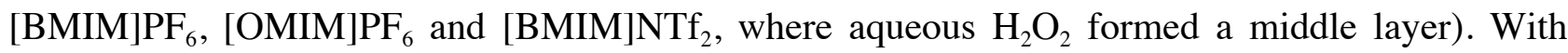
TBHP, only a biphasic system formed for each IL tested. As mentioned earlier, during ODS the catalyst I was distributed at the middle layer of the solvent mixture, while the corresponding sulfones were completely extracted by ILs. At the end of the reaction, only residual DBT was detected (when still present) in the recovered hydrocarbon phase. As showed in Fig. 3, in comparison with $\mathrm{H}_{2} \mathrm{O}_{2}$, TBHP showed to be only moderately active for all used ILs, with conversion of DBT ranging from $20 \%$ to $62 \%$. The lower activity observed with TBHP can be attributed to several reasons, among others the occurrence of different reaction pathways operative for each type of oxidant and ILs, and has been already observed using [BMIM] $\mathrm{PF}_{6}$ [21]. With $\mathrm{H}_{2} \mathrm{O}_{2}$, all ILs gave a comparable behavior, affording a complete extraction of the corresponding sulfone, after only 2 hours. Actually, analyzing in detail the progress of the DBT oxidation, with $\mathrm{H}_{2} \mathrm{O}_{2}$ (Figure 4), it can be observed that in most cases the complete oxidation of s.m. was reached within the first hour, with the only relevant exception for [BMIM] $\mathrm{NTf}_{2}$, where about a $12 \%$ of s.m. remained to be converted after 1 hour (see Fig. 4). 


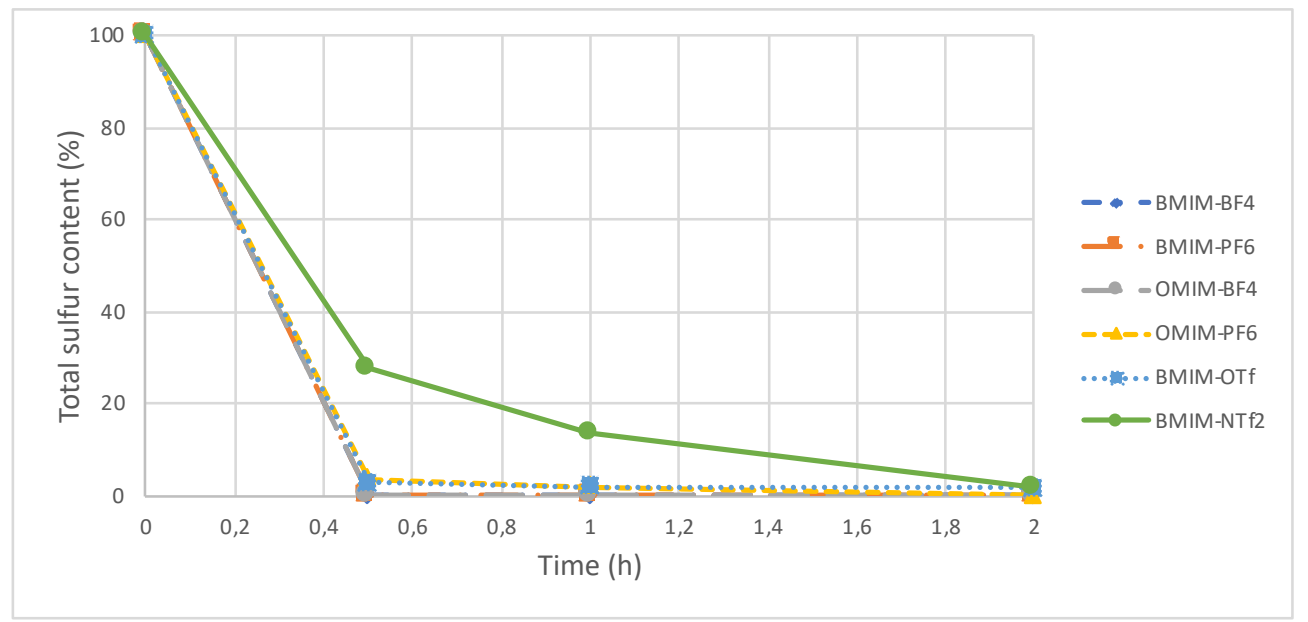

Figure 4. Details of the ECODS of DBT, with $\mathrm{H}_{2} \mathrm{O}_{2}$ and catalyst I in six different $n$-octane/IL mixtures

From literature, it is known that the ability of 1-alkyl-3-methylimidazolium ILs to increase the extraction of $\mathrm{S}$ containing aromatic substrates is related to the effective occurrence of C2$\mathrm{H}$ (imidazolium)--- $\pi$ bonds interactions, thus increasing with the increase of $\pi$-density and decreasing with the degree of alkyl substituents on the ring of sulfur substrates. Moreover, the percentage of the extraction may be also related to the strength of $\mathrm{C} 2-\mathrm{H}$ imidazolium hydrogen bond with the anion, in ILs, following the order $\mathrm{BF}_{4}>\mathrm{OTf}>\mathrm{PF}_{6}>\mathrm{NTf}_{2}$. Thus, in order to increase the efficiency of the extraction, the sulfur substrates have to disrupt hydrogen bonds formed between the $\mathrm{C} 2-\mathrm{H}$ of the imidazolium cation and the anion of the IL (ion pair effect). The restructuring process is primarily driven by the interaction of sulfur derivative with the imidazolium cations of the ILs; moreover, the maximum absorption capacity of sulfur substrate, by ILs, is primarily determined by the size and structure of both ILs cation and anion [22]. Regarding the effect deriving from the length of the chain of the imidazolium alkyl substituent, in the IL cation, it seems that its increasing may increase the extraction percentage of organic sulfur compounds $[23,12 b]$. Just to confirm whether or not these evidences could have a role, under our conditions, we decided to select a few ILs, namely [BMIM]BF , $_{4}[\mathrm{OMIM}] \mathrm{BF}_{4}$ and [BMIM]OTf, [12c,24], and evaluate their effective role in the ECODS process for several types of recalcitrant benzothiophene derivatives, among the most representative contained in a real diesel fuel, namely DMDBT, BT, 2Me-BT and 3Me-BT. The selection of imidazolium ILs, has been made based on their water miscibility (considering the use of aq. $\mathrm{H}_{2} \mathrm{O}_{2}$ ), and also taking into consideration both the different relative dimensions of the cation (BMIM vs OMIM) and the different potential role for the sulfones extraction ability, in terms of strength and compactness of the corresponding IL ion pair, due to the diverse anion $\left(\mathrm{BF}_{4}^{-}\right.$vs. TfO-). 
All sulfur substrates were studied under the same conditions, namely $0.1 \mathrm{mmol}$ of s.m. in a 5:1 (v/v) mixture of $n$-octane/IL (1.2 mL), $1.0 \mathrm{~mol} \%$ of catalyst $\mathbf{I}$, an excess of $\mathrm{H}_{2} \mathrm{O}_{2}$ (4.0 equiv.), at $50{ }^{\circ} \mathrm{C}$, for $3 \mathrm{~h}$, under stirring. In order to avoid the fast and unfruitful decomposition of $\mathrm{H}_{2} \mathrm{O}_{2}$, after the initial addition (2.0 equiv.) the excess was added portionwise after, respectively, $1 \mathrm{~h}$ (1.0 equiv.) and $2 \mathrm{~h}$ (1.0 equiv.).
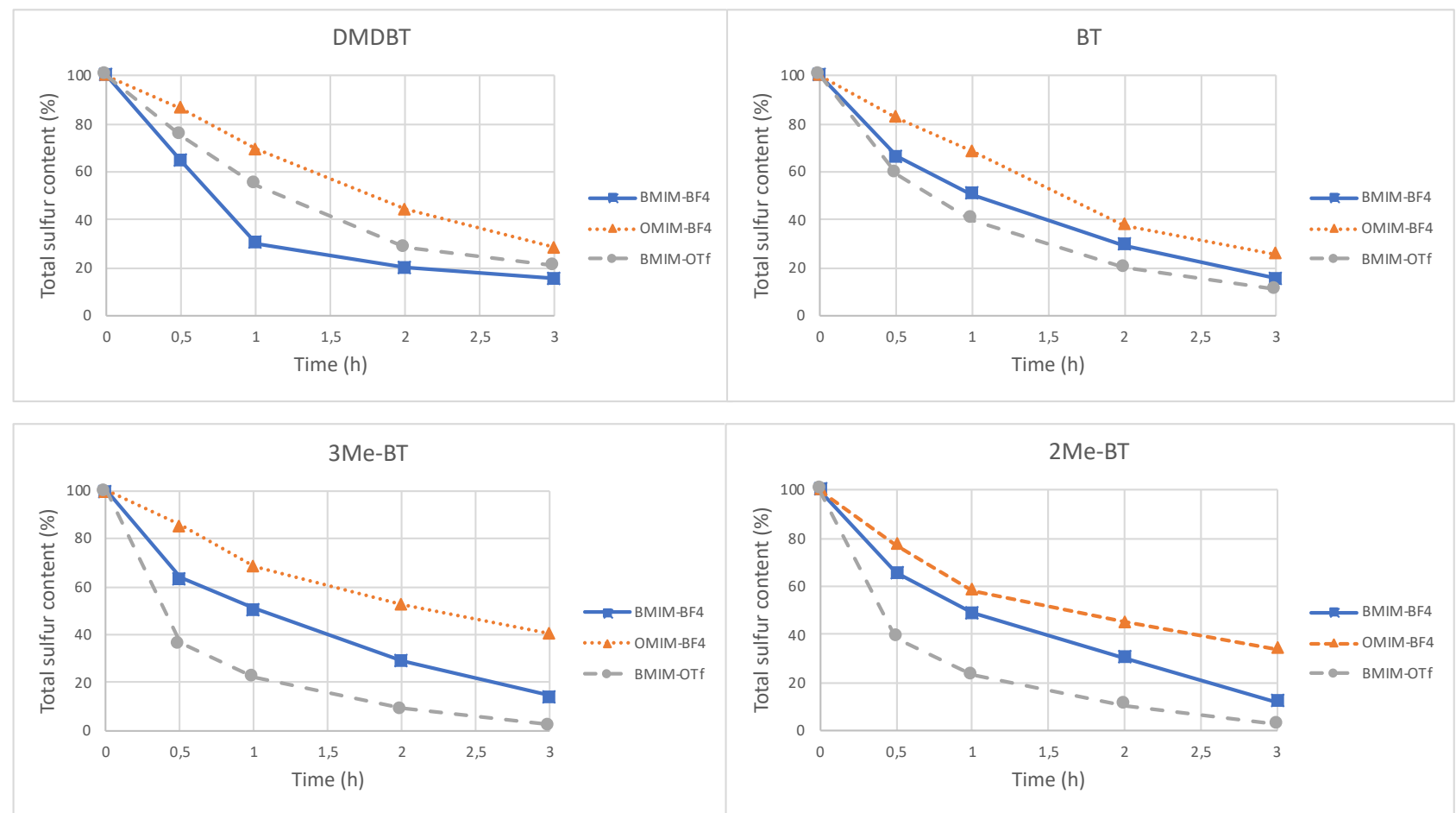

Figure 5. ECODS of DMDBT and BT (above), and 3Me-BT and 2Me-BT (below), with catalyst I and $\mathrm{H}_{2} \mathrm{O}_{2}$, for three different n-octane/IL mixtures.

As showed in Fig. 5, all sulfur substrates gave a very good conversion toward the corresponding sulfones, after 3 hours, with values ranging from a minimum of $60 \%$ for $3 \mathrm{Me}-\mathrm{BT}$ in $[\mathrm{OMIM}] \mathrm{BF}_{4}$, to quantitative oxidation and complete extraction for 3Me-BT and 2Me-BT in [BMIM]OTf. In terms of sulfur removal efficiency, relatively to the extraction of sulfones by ILs, we observed the

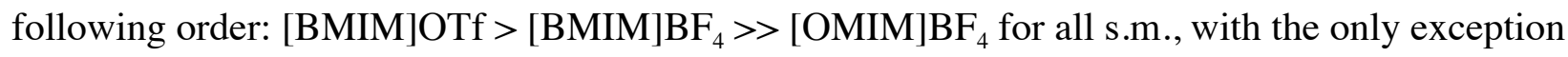
for DMDBT where [BMIM]BF 4 showed to act as the most efficient system in the sequence, although not by much. From these results, we can tentatively conclude that the supramolecular clusters formed between the cation $[\mathrm{BMIM}]^{+}$and anion, seems to be more prone to be disrupted by the sulfone when triflate (TfO-) is involved as anion, hence increasing the extraction efficiency [23]. The order of activity observed for the oxidation of s.m. in [BMIM]OTf, namely DBT $>2 \mathrm{Me}-\mathrm{BT} \geq$ $3 \mathrm{Me}-\mathrm{BT}>\mathrm{BT} \geq \mathrm{DMDBT}$, was slightly different from what observed previously for [BMIM]BF ${ }_{4}$ 
(see Fig. 1), therefore validating that several factors may affect simultaneously the efficiency of both the oxidation reaction and the extraction process of the sulfones.

\subsection{ODS of a model diesel}

The successive experiments were performed using a model diesel containing, simultaneously, equimolar amounts of all the above described sulfur compounds normally present in a real diesel, giving a total sulfur concentration of $2700 \mathrm{ppm}$ in $n$-octane. Each ECODS experiment for the model

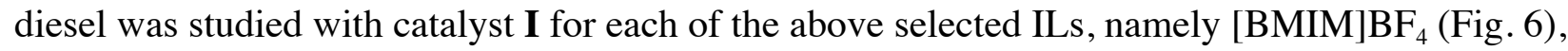
$\left[\mathrm{OMIM}_{\mathrm{B}} \mathrm{BF}_{4}\right.$ (Fig. 7) and [BMIM]OTf (Fig. 8), also with the aim to evaluate the occurrence, if any, of synergic effects between the diverse s.m., affecting the overall process. In this case, we slightly increased the overall reaction time (6 hours) and the excess of $\mathrm{H}_{2} \mathrm{O}_{2}$ (up to 5.0 equiv.) in order to increase the desulfurization efficiency, otherwise working under the same conditions. Also in this situation, after the initial addition of 2.0 equiv. of oxidant, the latter excess has been added portionwise after $1 \mathrm{~h}, 2 \mathrm{~h}$ and $3 \mathrm{~h}$, respectively. The progress of the oxidation was periodically monitored by GC-FID analysis (after $0.5,1,2,3$ and 4 hours, respectively). If we compare the kinetic of DBT oxidation, i.e. the most reactive substrate, we can observe that depending on the IL used it showed a different behavior. Indeed, after the first 30 minutes, in $[\mathrm{BMIM}] \mathrm{BF}_{4}$ the residual amount of sulfur to be oxidized was only $11 \%$, while we observed a residual amount of, respectively, $42 \%$ and $58 \%$ in $[\mathrm{OMIM}] \mathrm{BF}_{4}$ and [BMIM] $\mathrm{OTf}$, being the complete oxidation reached

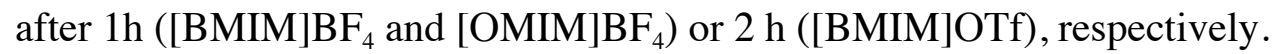

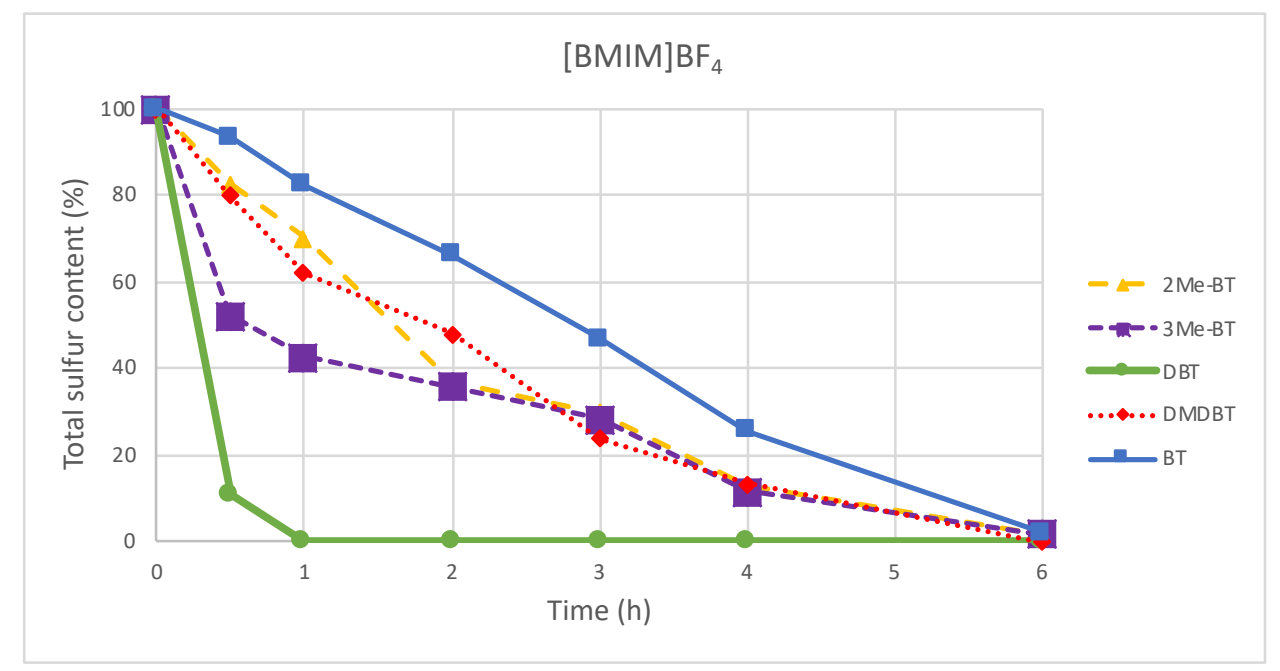

Figure 6. ECODS of a model diesel $\left(\mathrm{S}_{\text {tot }}=2700 \mathrm{ppm}\right)$ vs. reaction time, in $[\mathrm{BMIM}] \mathrm{BF}_{4}$. Conditions: $\mathrm{T}=50{ }^{\circ} \mathrm{C}, \mathrm{S} / \mathrm{H}_{2} \mathrm{O}_{2}$ $=1 / 5$ (molar ratio); $1.0 \mathrm{~mol} \%$ of catalyst $\mathbf{I}, 1.2 \mathrm{~mL}$ of $n$-octane $/ \mathrm{IL}=5: 1$. 


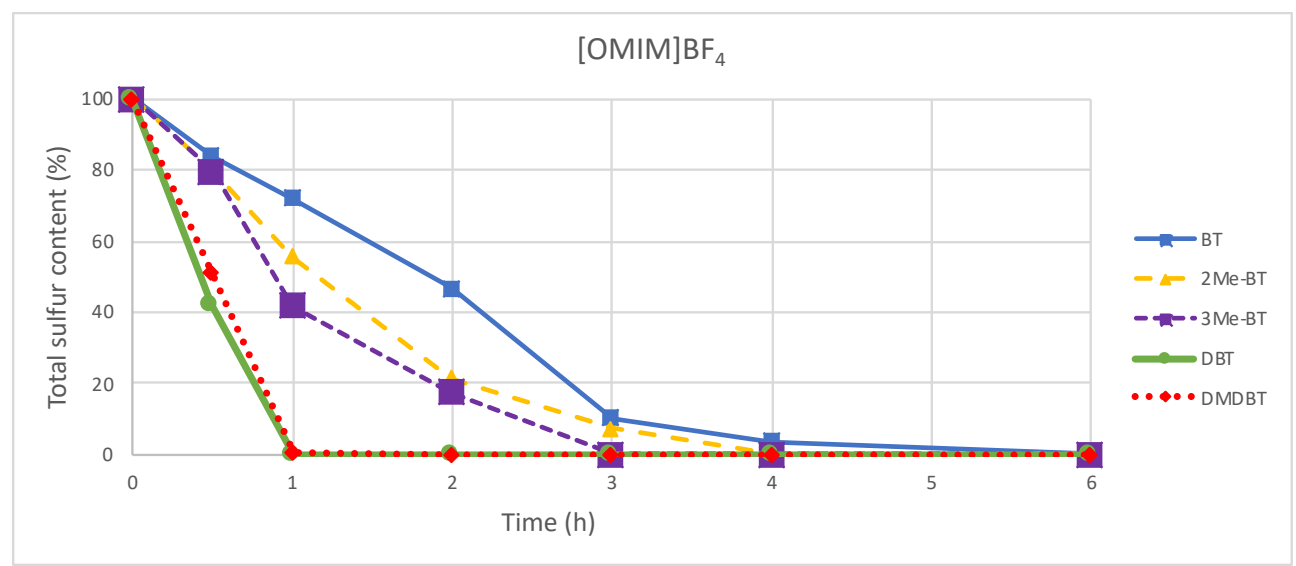

Figure 7. ECODS of a model diesel $\left(\mathrm{S}_{\text {tot }}=2700 \mathrm{ppm}\right)$ vs. reaction time, in $[\mathrm{OMIM}] \mathrm{BF}_{4} \cdot \mathrm{Conditions} \mathrm{T}=50^{\circ} \mathrm{C}, \mathrm{S} / \mathrm{H}_{2} \mathrm{O}_{2}$ $=1 / 5$ (molar ratio); $1.0 \mathrm{~mol} \%$ of catalyst $\mathbf{I}, 1.2 \mathrm{~mL}$ of $n$-octane $/ \mathrm{IL}=5: 1$.

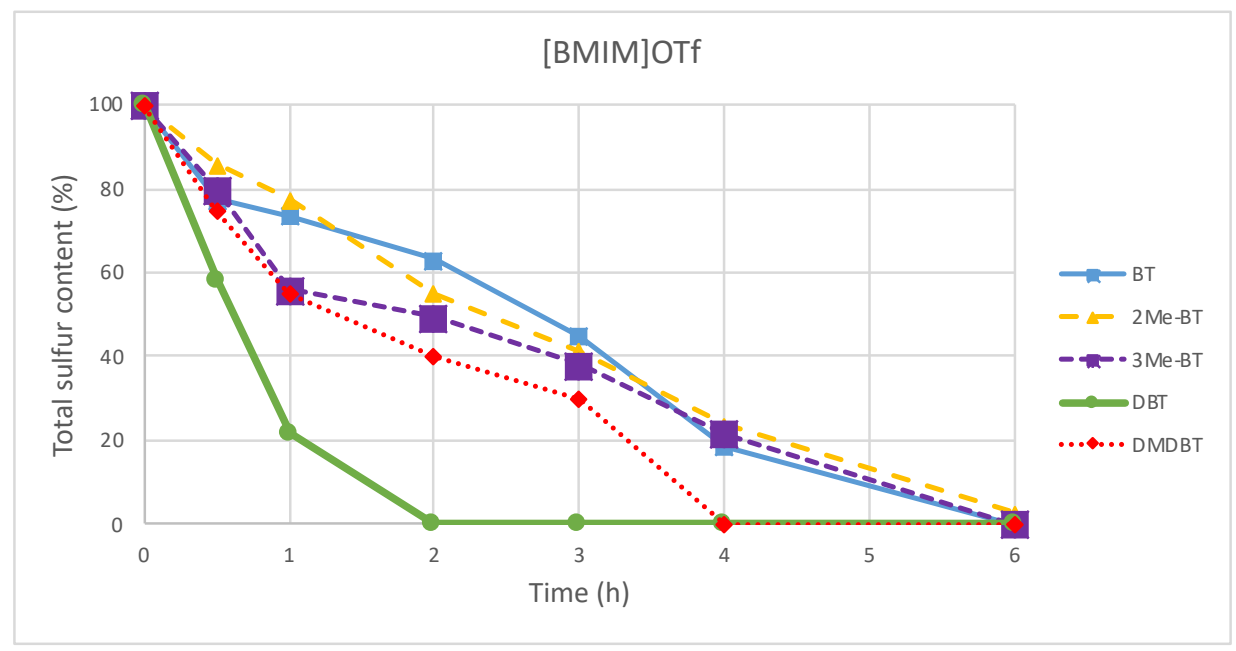

Figure 8. ECODS of a model diesel $\left(\mathrm{S}_{\text {tot }}=2700 \mathrm{ppm}\right)$ vs. reaction time, in [BMIM]OTf. Conditions: $\mathrm{T}=50{ }^{\circ} \mathrm{C}, \mathrm{S} / \mathrm{H}_{2} \mathrm{O}_{2}$ $=1 / 5$ (molar ratio); $1.0 \mathrm{~mol} \%$ of catalyst $\mathbf{I}, 1.2 \mathrm{~mL}$ of $n$-octane $/ \mathrm{IL}=5: 1$.

A different behavior was detected when we studied the oxidation of DBT alone for the different ILs (see Fig. 4), where the almost quantitative conversion toward the sulfone was obtained during the first 30 minutes, with the only exception given by the $n$-octane/[BMIM]OTf system. These results showed that, for the model diesel, the presence of negative effects introducing a competition, among the sulfurated substrates for the catalytic oxidation of sulfur, cannot be excluded. 
Analyzing the percentage of oxidized substrates, depending on reaction time, it is evident that the best score was obtained with [OMIM] $\mathrm{BF}_{4}$ (Fig. 7) where for four over five s.m. the residual amount of substrate was about only $20 \%$ (being the BT the less reactive), after 2 hours. Interestingly, the kinetics in [BMIM]OTf were significantly lower, for the model diesel, after $2 \mathrm{~h}$ of reaction (Fig 8 , residual S amount between 40 to $65 \%$ with the exception of DBT) with respect to that observed, at the same time, in the oxidation of each s.m. alone (Fig. 4 and 5). It may be invoked that competitive effects between the different oxidized sulfones might negatively affect their partition into the ionic liquid phase. Anyway, all sulfur substrates were quantitatively converted to their corresponding sulfones and removed from the model diesel, within 6 hours, hence confirming the efficacy of ECODS process based on the use of the above described imidazolium ILs along with the catalyst $\mathbf{I}$.

\subsection{Recyclability experiments}

The recyclability of the catalytic system was studied for the model diesel above described, with catalyst I and [OMIM]BF${ }_{4}$, for 6 hours. It should be emphasized that molybdenum catalyst, dispersed at the interphase, retains its activity thus allowing its recyclability. After the first cycle, the model diesel phase was recovered and analyzed by GC-FID to evaluate its $\mathrm{S}$ content. The residue of water was eliminated by evaporation under reduced pressure. Then, new fresh portions of both model diesel and $\mathrm{H}_{2} \mathrm{O}_{2}$ were added to the recycled IL phase containing the entrapped catalyst, and a new cycle started. Since the first cycle, trace of a white precipitated was detected in the mixture, which increased in the course of the next recycling steps. It was removed and analyzed, by FTIR, after the fifth cycle, showing the characteristically absorption bands of sulfone group [25]. As showed in Table 1, very interestingly, the catalytic system retained, at all unmodified, the original activity at least for the first three cycles, showing only a very slight decreasing of activity after the third and fourth recycle.

Table 1. Recyclability of the catalytic system for the ECODS of the model diesel with catalyst $\mathbf{I}$ and $[\mathrm{OMIM}] \mathrm{BF}_{4}$, after 6 hours.

\begin{tabular}{cccccc}
\hline Cycle & \multicolumn{5}{c}{ Total Sulfur Conversion (\%) } \\
\hline & DBT & BT & 2Me-BT & 3Me-BT & DMDBT \\
\hline 1 & 99 & 99 & 99 & 99 & 99 \\
\hline 2 & 99 & 99 & 99 & 99 & 99 \\
\hline 3 & 99 & 99 & 99 & 99 & 99 \\
\hline 4 & 98 & 93 & 95 & 96 & 95 \\
\hline 5 & 97 & 92 & 94 & 95 & 93 \\
\hline
\end{tabular}




\subsection{Catalytic ECODS of the model diesel under $M W$}

Finally, with the idea to further investigate the potentiality of our catalytic system, we decided to exploit the microwave irradiation as energy source for the ECODS, to optimize the reaction time as much as possible. On the other hand, the combination of microwave irradiation, as energy source, with ionic liquids, either as solvents or catalysts, has been largely investigated, in recent years [26]. In addition, microwave heating technologies have been proved to be efficient in desulfurization of petroleum oils [27]. It seems clear that the operative conditions are less harsh and treatment time shorter by using microwave heating than conventional sources. Anyway, despite these encouraging premises, only a few articles were so far published concerning the use of microwave assisted conditions for the study of catalytic ODS processes, aiming at reducing the sulfur content in different types of fuel [28].

For the procedure, we optimized the following conditions for the MW reactor: running time $=28$ min., preceded by a prestirring of $2 \mathrm{~min}$, for every $\mathrm{MW}$ cycle; $\mathrm{T}=90^{\circ} \mathrm{C}$, Power Output average $=$ $200 \mathrm{~W}$, stir rate $=720 \mathrm{rpm}$, amount of total S for the model diesel phase $=2700 \mathrm{ppm}$, otherwise working under similar optimized conditions above reported. Each experiment has been performed by using the appropriate MW glass sealed vials $(0.5-2.0 \mathrm{~mL})$. In the case of $[\mathrm{BMIM}] \mathrm{BF}_{4}$ and [OMIM] $\mathrm{BF}_{4}$ four successive cycles $(28+2 \mathrm{~min}$.) were necessary to obtain the full sulfur removal for a total reaction time of $2 \mathrm{~h}$ (see Fig. 9 and 10). If we analyze the experimental results by comparing the substrates activity, with respect to the different ILs, the following main conclusions

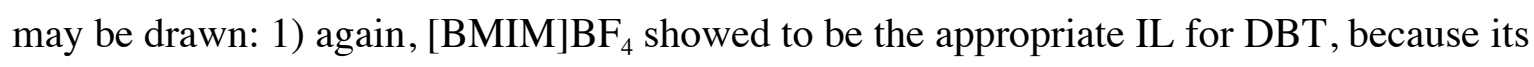
complete oxidation and extraction was achieved after the $1^{\text {st }}$ cycle, instead being it able to better differentiate the reactivity of the other substrates during the remaining three cycles (Fig. 9); 2) $[\mathrm{OMIM}] \mathrm{BF}_{4}$ confirmed once again to be the most effective IL, due to the fact that almost complete sulfur removal (for three over five s.m.) was reached after the $1^{\text {st }} \mathrm{MW}$ cycle, being BT and DMDBT the less reactive (Fig. 10). Quite interestingly, in the case of [BMIM]OTf, only two MW cycles were necessary to obtain the complete sulfur removal from the model diesel, after a total reaction time of only 1 hour, being also in this case DBT the most active substrate (Fig. 11). The latter result confirms the ability of [BMIM]OTf as valuable extracting medium of sulfur containing substrates, like DBT, as previously observed in literature [24]. Anyway, the relevant role due to the positive effects deriving from the microwave energy source, cannot be ruled out. 


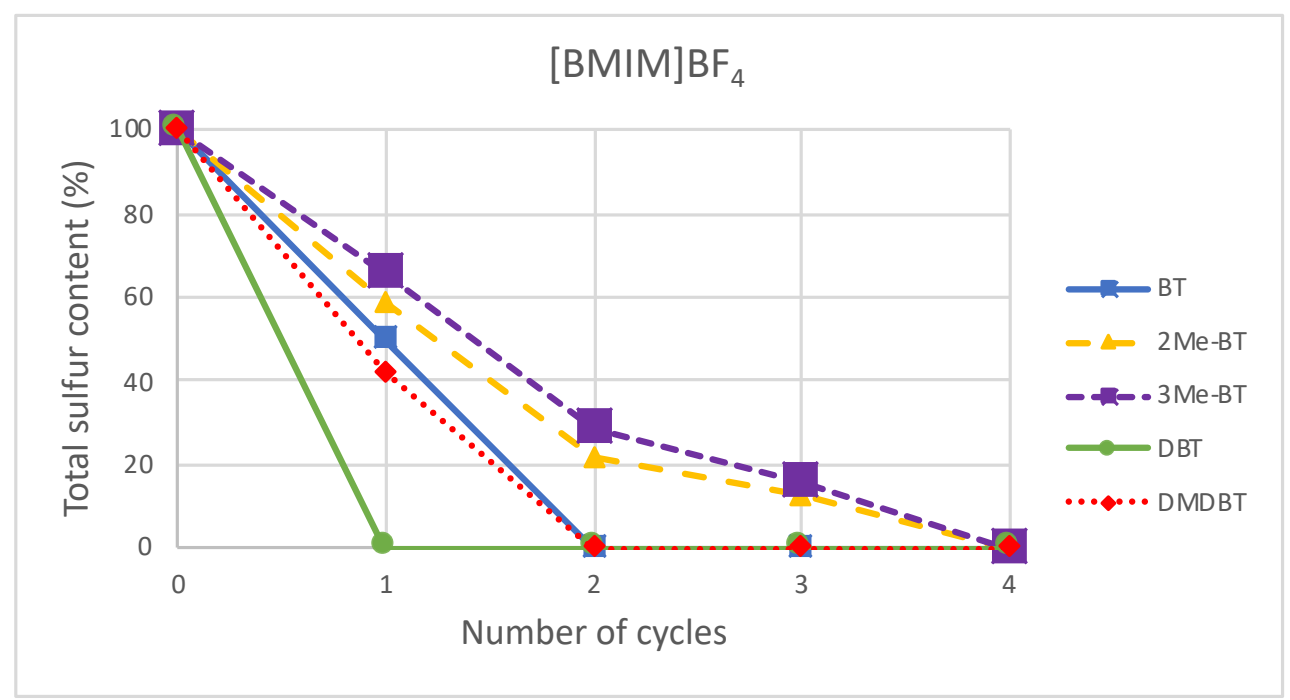

Figure 9. ECODS of a model diesel $\left(\mathrm{S}_{\mathrm{tot}}=2700 \mathrm{ppm}\right)$ in $[\mathrm{BMIM}] \mathrm{BF}_{4}$, with catalyst $\mathbf{I}$ and $\mathrm{H}_{2} \mathrm{O}_{2}$, under MW conditions

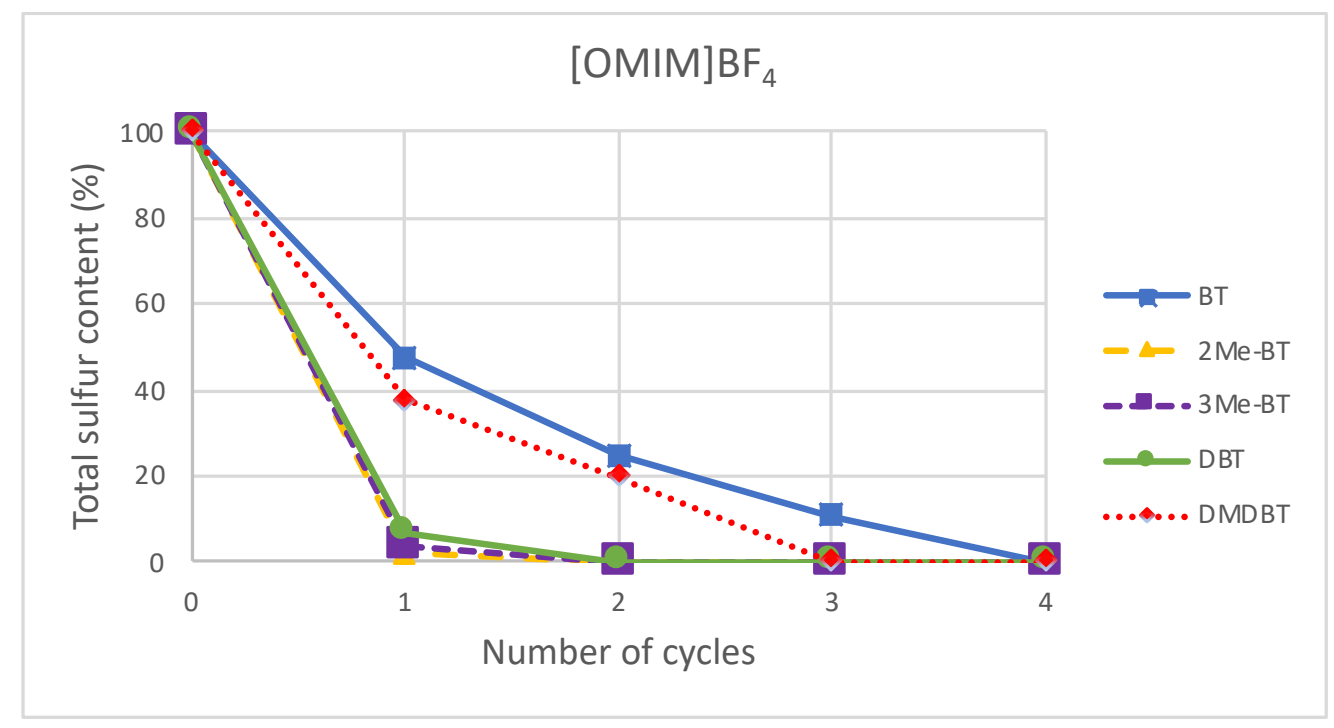

Figure 10. ECODS of a model diesel $\left(\mathrm{S}_{\text {tot }}=2700 \mathrm{ppm}\right)$ in $[\mathrm{OMIM}] \mathrm{BF} F_{4}$, with catalyst $\mathbf{I}$ and $\mathrm{H}_{2} \mathrm{O}_{2}$, under $\mathrm{MW}$ conditions 


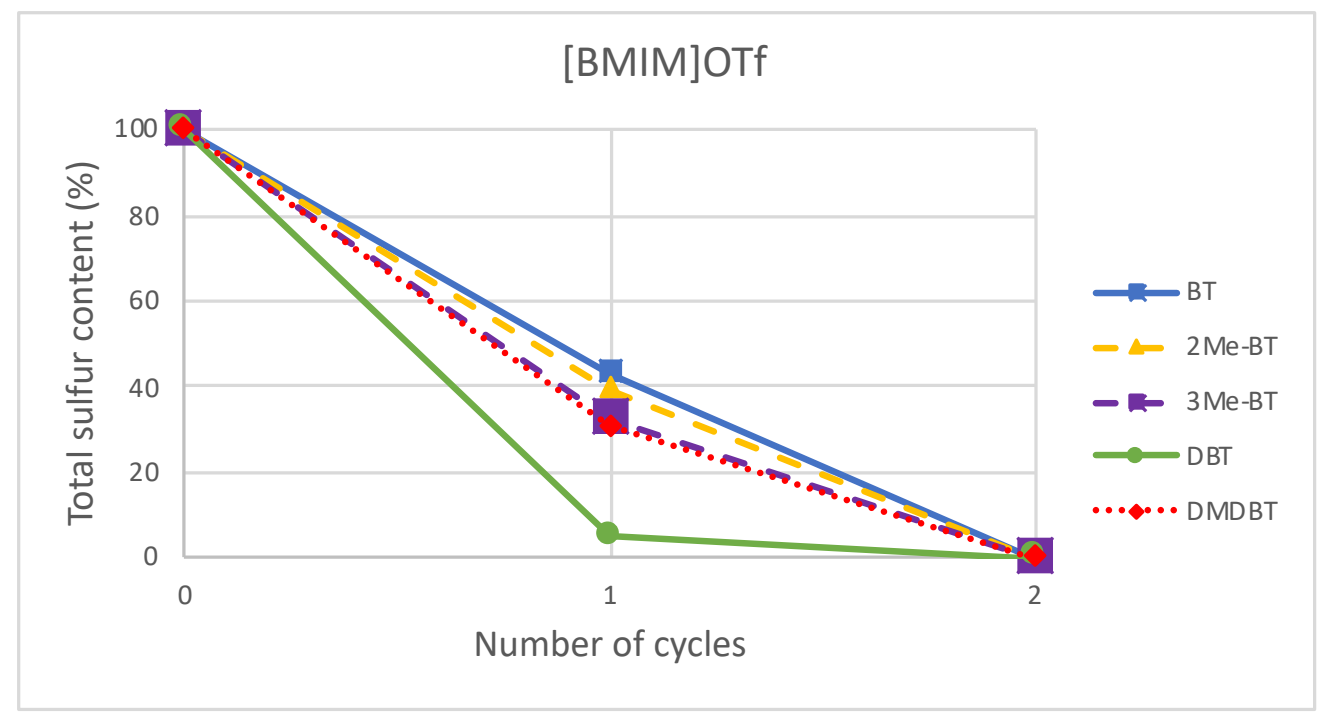

Figure 11. ECODS of a model diesel $\left(\mathrm{S}_{\mathrm{tot}}=2700 \mathrm{ppm}\right)$ in [BMIM]OTf, with catalyst $\mathbf{I}$ and $\mathrm{H}_{2} \mathrm{O}_{2}$, under MW conditions.

\section{Conclusions}

A new application of the ECODS methodology for a model diesel based on the extraction of sulfone derivatives using a selection of common imidazolium ionic liquids, and catalytic oxidation with a series of cis-dioxomolybdenum(VI) $\alpha$-amino acid containing compounds $\mathbf{I}-\mathbf{V}$, has been developed under heterogeneous conditions. The sulfur removal from the model diesel phase, formed by several recalcitrant sulfurated substrates like BT, DMDBT, DBT, 2Me-Bt and 3Me-Bt, for a total S loading of $2700 \mathrm{ppm}$, reached quantitative values within 6 hours, under mild thermal heating. More surprisingly, when ECODS process was studied under MW assisted conditions, the same quantitative elimination of sulfur containing substrates was reached after only 2 hours, in the case of $[\mathrm{BMIM}] \mathrm{BF}_{4}$ and $[\mathrm{OMIM}] \mathrm{BF}_{4}$ or, even after $1 \mathrm{~h}$ when using [BMIM]OTf. The quite heterogeneous cis-dioxomolybdenum(VI) catalytic precursor I, dispersed at the interphase, acted as a very stable system, retaining its catalytic activity at least for the first three cycles, while showing only a very slight decreasing of activity after the third and fourth recycling step. Once again, the ionic liquid played a fundamental role not only as extractant and reaction medium but also as stabilizing agent of both the oxidant and the catalytic system, in the reaction medium [12e]. Anyway, the remarkable advantage of ECODS process over the desulfurization by mere solvent extraction promoted by ILs highlights the indispensability and versatility of a robust catalyst such as those based on oxomolybdenum(VI) complexes and validates their large use on this type of processes. Although, several studies have been reported, so far, concerning the use of transition metal based complexes for ECODS reactions, only a few have highlighted the potentially 
interesting role related to the use of microwave assisted technology. Thus, this work represents a valid contribution in this direction. Further studies devoted to evaluate the full applicability of our catalyst precursors for ECODS processes applied to real diesel samples, will be planned. In addition, the selection of proper ILs able to avoid the removing of aromatics and olefins, i.e. key compounds to prevent the lowering of diesel quality, will be an important target.

\section{Acknowledgements}

The Universities of L'Aquila and Pisa are gratefully acknowledged for financial support.

\section{References}

[1] (a) E.B. Fox, Z.-W. Liu, Z.-T. Liu, Energy Fuels 27 (2013) 6335-6338.

(b) F.T. Li, R.H. Liu, J.H. Wen, D.S. Zhao, Z.M. Sun, Y. Liu, Green Chem. 11 (2009) 883-888.

[2] V.C. Srivastava, RSC Adv. 2 (2012) 759-783.

[3] C. Song, Catal. Today 86 (2003) 211-263.

[4] (a) A. A. Nuhu, Rev. Environ. Sci. Biotechnol. 12 (2013) 9-23.

(b) Y. S. Chi, C. P. Li, Q. Z Jiao, Q. S. Liu, P. F. Yan, X. M. Liu, W.-B. Urs, Green Chem. 13 (2011) 1224-1229.

(c) J. L. Wang, D. S. Zhao, K. X. Li, Energy Fuels 24 (2010) 2527-2529.

(d) M. Soleimani, A. Bassi, A. Margaritis, Biotechnol. Adv. 25 (2007) 570-596.

[5] R. Saladino, G. Botta, M. Crucianelli, Advances in Nanostrostructured Transition Metal Catalysis Applied to Oxidative Desulfurization Processes: Nanotechnology Applied to ODS Processes, in Applying Nanotechnology to the Desulfurization Process in Petroleum Engineering, T. A. Saleh (Ed.), IGIGlobal, USA, 2016, pp. 180-216; DOI: 10.4018/978-1-46669545-0.ch006.

[6] (a) C. Dai, J. Zhang, C. Huang, Z. Lei, Chem. Rev. 117 (2017) 6929-6983.

(b) H. Zhao, G. A. Baker, Front. Chem. Sci. Eng. 9 (2015) 262-279.

(c) A. Bösmann, L. Datsevich, A. Jess, A. Lauter, C. Schmitz, P. Wasserscheid, Chem. Commun., (2001) 2494-2495.

[7] (a) D. Betz, P. Altmann, M. Cokoja, W. A. Herrmann, F. E. Kuihn, Coord. Chem. Rev. 255 (2011) 1518-1540.

(b) J. W. Lee, J. Y. Shin, Y. S. Chun, H. B. Jang, C. E. Song, S.-G. Lee, Acc. Chem. Res. 43 (2010) 985-994.

[8] (a) J.-K. Li, Y.-Q. Xu, C.-W. Hu, Inorg. Chem. Commun. 60 (2015) 12-14. 
(b) W. Zhang, H. Zhang, J. Xiao, Z. Zhao, M. Yu, Z. Li, Green Chem. 16 (2014) 211-220.

[9] (a) B. Martin, J. Sedelmeier, A. Bouisseau, P. Fernandez-Rodriguez, J. Haber, F. Kleinbeck, S. Kamptmann, F. Susanne, P. Hoehn, M. Lanz, L. Pellegatti, F. Venturoni, J. Robertson, M. C. Willis, B. Schenkel, Green Chem. 19 (2017) 1439-1448.

(b) M. Beller, Adv. Synth. Catal. 346 (2004) 107-108.

[10] see among others: (a) F. Mirante, L. Dias, M. Silva, S. O. Ribeiro, M. C. Corvo, B. de Castro, C. M. Granadeiro, S. S. Balula, Catal. Commun. 104 (2018) 1-8.

(b) C.M. Granadeiro, L.S. Nogueira, D. Julião, F. Mirante, D. Ananias, S.S. Balula, L. CunhaSilva, Catal. Sci. Technol. 6 (2016) 1515-1522.

(c) S.O. Ribeiro, D. Julião, L. Cunha-Silva, V.F. Domingues, R. Valença, J.C. Ribeiro, B. de Castro, S.S. Balula, Fuel 166 (2016) 268-275.

(d) C.M. Granadeiro, S.O. Ribeiro, M. Karmaoui, R. Valença, J.C. Ribeiro, B. de Castro, L. Cunha-Silva, S.S. Balula, Chem. Commun. 51 (2015) 13818-13821.

(e) D. Julião, A.C. Gomes, M. Pillinger, L. Cunha-Silva, B. De Castro, I.S. Gonçalves, S.S. Balula, Fuel Proc. Technol. 131 (2015) 78-86.

[11] (a) C.G. Piscopo, J. Tochtermann, M. Schwarzer, D. Boskovic, R. Maggi, G. Maestri, S. Loebbecke, React. Chem. Eng. 3 (2018) 13-16.

(b) A.T. Shah, B. Li, Z.E.A. Abdalla, J. Colloid Interface Sci. 336 (2009) 707-711.

[12] (a) D. Piccinino, I. Abdalghani, G. Botta, M. Crucianelli, M. Passacantando, M. L. Di Vacri, R. Saladino, Appl. Catal. B, 200 (2017) 392-401.

(b) D. Julião, A.C. Gomes, M. Pillinger, R. Valença, J.C. Ribeiro, I.S. Gonçalves, S.S. Balula, Dalton Trans. 45 (2016) 15242-15248.

(c) M. Zhou, W. Meng, Y.Li, Q. Wang, X. Li, S. Zang, Energy Fuels 28 (2014) 516-521.

(d) A. Mota, N. Butenko, J.P. Hallett, I. Correia, Catal. Today 196 (2012) 119-125.

(e) W. Zhu, H.Li, Q. Gu, P. Wu, G. Zhu, Y. Yan, G. Chen, J. Mol. Catal. A 336 (2011) 16-22.

(f) W. Zhu, H.Li, X. Jiang, Y. Yan, J. Lu, J. Xia, Energy Fuels 21 (2007) 2514-2516.

[13] M. Amini, M.M. Haghdoost, M. Bagherzadeh, Coord. Chem. Rev. 257 (2013)1093-1121.

[14] R.D. Chakravarthy, V. Ramkumar, D.K. Chand, Green Chem. 16 (2014) 2190-2196.

[15] L. Biancalana, M. Bortoluzzi, C. Forte, F. Marchetti, G. Pampaloni, RSC Adv. 5 (2015) 90109018

[16] H. Lü, C. Deng, W. Ren, X. Yang, Fuel Process. Technol. 119 (2014) 87-91.

[17] I. Abdalghani, L. Biancalana, M. Aschi, G. Pampaloni, F. Marchetti, M. Crucianelli, Molecular Catalysis 446 (2018) 39-48.

[18] (a) W. Zhu, W. Huang, H. Li, M. Zhang, W. Jiang, G. Chen, C. Han, Fuel Proc. Technol. 92 
(2011) 1842-1848.

(b) S. Otsuki, T. Nonaka, N. Takashima, W. Qian, A. Ishihara, T. Imai, T. Kabe, Energy Fuels 14 (2000) 1232-1239.

[19] (a) S.B. Abdullah, H.A. Aziz, Z. Man, Ionic Liquids for Desulphurization: A Review, in Recent Advances in Ionic Liquids, M. Rahman, (Ed.), 2018, IntechOpen, DOI:

10.5772/intechopen.79281.

(b) R. Abro, A.A. Abdeltawab, S.S. Al-Deyab, G. Yu, A.B. Qazi, S. Gao, X. Chen, RSC Adv. 4 (2014) 35302-35317.

(c) X. Chen, S. Yuan, A.A. Abdeltawab, S.S. Al-Deyab, J. Zhang, L. Yu, G. Yu, Sep. Purif. Technol. 133 (2014) 187-193.

[20] F.M. Collins, A.R. Lucy, C. Sharp, J. Mol. Catal. A Chem. 117 (1997) 397-403.

[21] D. Julião, A.C. Gomes, M. Pillinger, R. Valença, J.C. Ribeiro, I.S. Gonçalves, S.S. Balula, Appl. Catal. B 230 (2018) 177-183.

[22] A.-L. Revelli, F. Mutelet, J.-N. Jaubert, J. Phys. Chem. B 114 (2010) 4600-4608.

[23] C.C. Cassol, A.P. Umpierre, G. Ebeling, B. Ferrera, S.S.X. Chiaro, J. Dupont, Int. J. Mol. Sci. 8 (2007) 593-605.

[24] K. Kedra-Krolik, F. Mutelet, J.-C. Moïse, J.-N. Jaubert, Energy Fuels 25 (2011) 1559-1565.

[25] S. Otsuki, T. Nonaka, N. Takashima, W.H. Qian, A. Ishihara, T. Imai, T. Kabe, Energy Fuels 14 (2000) 1232-1239.

[26] B. Floris, F. Sabuzi, P. Galloni, V. Conte, Catalysts 7 (2017) 261: doi:10.3390/catal7090261.

[27] H. Shang, H. Zhang, W. Du, Z. Liu, J. Ind. Eng. Chem. 19 (2013) 1426-1432.

[28] (a) S. Mesdour, C. Lekbir, L. Doumandji, B. Hamada, J. Sulfur Chem. (2017) https://doi.org/10.1080/17415993.2017.1304550.

(b) R. Lu, J. Yang, X. Xu, J. Gao, Pet. Sci. Technol. 27 (2009) 1789-1799.

(c) Q. Fei, J. Yang, Xu X, J. Gao, ibid., 27 (2009) 764-775.

(d) N. E. Leadbeater, M. R. Khan, Energy Fuels 22 (2008) 1836-1839.

(e) A. D. Mohammed, A. G. Isah, M. Umaru, Int. J. Energy Environ. 3 (2012) 949-960. 